\title{
Implementation aspects in DFT modulated filter bank transceivers for cognitive radio
}

\author{
Nour Mansour, Dirk Dahlhaus \\ Communications Laboratory, University of Kassel, Kassel, Germany
}

Email address:

mansour@uni-kassel.de (N. Mansour),dahlhaus@uni-kassel.de (D. Dahlhaus)

To cite this article:

Nour Mansour, Dirk Dahlhaus. Implementation Aspects in DFT Modulated Filter Bank Transceivers for Cognitive Radio. International Journal of Wireless Communications and Mobile Computing. Special Issue: 5G Wireless Communication Systems.

Vol. 2, No. 4-1, 2014, pp. 1-10. doi: 10.11648/j.wcmc.s.2014020401.11

\begin{abstract}
Discrete Fourier transform (DFT) modulated filter banks (FBs) are considered as strong tools used to implement both dynamic spectrum access and spectrum sensing in cognitive radio (CR) systems. High time-frequency (TF) resolution for spectral estimation and effective spectrum access with low complexity transceivers are the basic objectives in CR systems. However, the limitations of self-interference in DFT FBs as well as a primary user interference increase the overall transceiver complexity. In this paper, we design DFT modulated FBs which take into account the aforementioned contradicting requirements of high resolution capabilities, efficient spectrum access and affordable implementation effort for an additive white Gaussian channel. Four simple designs are presented and their performance are investigated and compared for a CR system with basic transmission parameters resembling those of IEEE 802.11g.
\end{abstract}

Keywords: Cognitive Radio, Filter Banks, Spectrum Access, Spectrum Sensing, Intersymbol Interference, Gabor System

\section{Introduction}

Intensive research activities are increasing rapidly in the field of cognitive radio (CR) due to the importance of the wireless spectrum in radio communications. To exploit this resource, unused slots of the spectrum by so-called prioritized primary users (PUs) are detected to be used by so-called secondary users (SUs). The SUs are allowed to use idle spectral resources given that the resulting interference experienced by the PU is limited, e.g. in the framework of underlay, overlay or interweave CR systems [1].

Spectrum sensing (SSE) [2], [3] requires good time-frequency (TF) resolution of the employed spectral estimation scheme to allow for subsequent spectrum access (SAC). The latter in turn benefits from good TF resolution of the employed waveforms due to limited availability of $\mathrm{TF}$ resources to be used by the SUs. The PU traffic patterns and the bit-error rate (BER) specifications of SU transmission together with spectral masks can be used to specify the conditions under which SSE and SAC schemes are designed. An efficient transceiver architecture for implementing SSE and SAC simultaneously should thus employ a common signal processing approach for both tasks. To this end, one option is to use discrete Fourier transform (DFT) modulated filter banks (FBs) where the spectrum under consideration is sensed by SU receivers (RXs) and potentially accessed by SU transmitters (TXs) in small portions of the TF plane.

A critical issue in the design of FBs is the TF correlation of FB output signals. Mutually orthogonal pulses in the TF plane used for inner products in the FBs can be constructed using DFT FBs which represent implementations of Gabor systems [4]. In case of mutually orthogonal pulses, zero intra-band and cross-band intersymbol interference (ISI) of SU signals as well as high accuracy for spectrum estimation can be achieved under certain conditions. Here, we can distinguish two cases. Firstly, one can consider so-called critically sampled DFT FBs [4]. Riesz bases in the Hilbert space of square-summable time signals $L^{2}(\mathbb{Z})$ with good TF concentration properties of the resulting pulse to be used in the FB, however, cannot be constructed according to the Balian-Low theorem [5]. Secondly, under-critically sampled FBs are described in [6]. In this case, the pulse TF concentration can be improved at the expense of a potential loss in transmission rate due to the incompleteness of the corresponding Gabor frame and the correspondingly missing perfect reconstruction property. In [6], a high implementation complexity is required to construct a pulse $\mathbf{g}=[g[0], \ldots, g[L-1]]^{\mathrm{T}}$ consisting of $L$ components in the time domain with $L \in \mathbb{N}$ in a so-called paraunitary 
over-critically sampled FB under the lattice being dual to the sought for Gabor system. Besides, a semidefinite programming to solve a semidefinite relaxation of the original optimization problem with a number of constraints [7] has to be implemented with an additional potential final rank reduction method.

In this paper, an alternative approach to the design of a DFT FB transceiver for SSE and SAC is proposed which aims at a system-specific approach taking into account SU BER specifications and PU interference and simultaneously can be implemented with limited complexity. Here, we consider for simplicity transmission over an additive white Gaussian noise (AWGN) channel. The main idea is to find a pulse $\mathbf{g}$ to be used in both SU TXs and SU RXs that result from constrained optimization. The objective function is the maximization of the pulse TF concentration where different concentration measures are applied. The constraints contain BER specifications of the SU taking into account intra-band and cross-band ISI from SU signals as well as third-party interference including the PU signal modeled as an additive white Gaussian process. Therefore, as long as the constraints are met for given values of the signal-to-noise ratio (SNR) and the interference based on the chosen objective function, no further optimization in the sense of achieving a global optimum of the objective function is required.

Four different constrained optimization approaches for optimizing the SSE performance and meeting a specific BER are presented [8]. The concentration of $\mathbf{g}$ at a certain point in the TF plane is measured using different dispersion metrics. We consider the minimization of a heuristic dispersion measure as well as the minimization of leakage in TF as well as separately in time or frequency based on the Rihaczek distribution. In addition to [8], we show a practical application of the design in the field of wireless local area networks (WLANs).

The paper is organized as follows. Sect. 2 describes the system model, the modulated DFT FBs used in the SU transceiver and the interference experienced by the SU RX. In Sect. 3, we discuss the transceiver design including the pulse optimization based on different leakage metrics for the four approaches. In Sect. 4, an overview of practical implementation aspects of the proposed transceiver is given based on the WLAN standard IEEE 802.11g. Sect. 5 shows pulses resulting from the optimization in Sect. 3 and their corresponding TF energy distribution. Furthermore, simulations are carried out to characterize the BER performance of the DFT FB transceiver in different interference environments. Finally, conclusions are drawn in Sect. 6.

Throughout the paper boldfaced characters are used for vectors and matrices. Furthermore, $\mathbf{E}\{\},.\|\cdot\|, \mathbf{X}^{\mathrm{T}}, a^{*}$, $\langle.,\rangle,.||,. \operatorname{Diag}[\mathbf{a}]$ and $\lceil$.$\rceil denote expectation, the$ Euclidean norm, transposition of matrix $\mathbf{X}$, complex conjugation of a complex number $a$, the inner product, the absolute value, a diagonal matrix composed of the elements of the vector $\mathbf{a}$ and the ceiling function, respectively.

\section{System Model}

\subsection{Frames and Filter Banks}

The authors in [4] derive the equivalence of DFT modulated FBs and Gabor frames in form of a relation between Gabor analysis/synthesis windows and the analysis/synthesis prototype filters of DFT modulated FBs. Here, we make use of this equivalence to describe the FBs.

The properties of a discrete-time signal $x[n]$ can be characterized with respect to time and frequency in the context of a DFT FB which contains a so-called prototype filter that is characterized by its discrete-time impulse response $g[n]$. We define a set $\left\{g_{\ell, k}[n]=g[n-\ell N] \mathrm{e}^{j 2 \pi(n-\ell N) k / K}:(\ell, k) \in \Lambda\right\}$ in $L^{2}(\mathbb{Z})$ as a Gabor system which constitutes a set of functions derived from $g[n]$ by time shifts $\ell N$ and frequency shifts $k / K$ with $(\ell, k) \in \Lambda$ and $\Lambda=\mathbb{Z} \times\{0, \ldots, K-1\}$ where $K$ and $N$ are positive integers chosen according to the system specifications. Using $\left\{g_{\ell, k}[n]\right\}$, two tasks can be accomplished. Firstly, under the assumption of a complete set $\left\{g_{\ell, k}[n]\right\}$, the channel state information can be acquired by SSE, where a received signal $y[n]$ is projected onto $g_{\ell, k}[n]$ by $y_{\ell, k}=\left\langle y[n], g_{\ell, k}[n]\right\rangle=\sum_{n \in \mathbb{Z}} y[n] g_{\ell, k}^{*}[n]$. Secondly, SAC can be done by transmitting data symbols $b^{(\ell, k)}$ at time $\ell$ and at frequency $k$ in form of a signal $b^{(\ell, k)} g_{\ell, k}[n]$ with $b^{(\ell, k)}$ being drawn from a suitable symbol alphabet. Here, we consider a $\pi / 4$-differential quaternary phase-shift keying ( $\pi / 4-$ DQPSK ) modulation. Consequently, both sensing and demodulation can be accomplished using the given receiver structure with identical analysis and synthesis windows of the Gabor system. As a result of the aforementioned equivalence, a Gabor system can be implemented using a modulated DFT FB with $K$ channels, where in each channel the signal is filtered and down-sampled by a factor $N$. Furthermore, the parameters $K$ and $N$ are chosen to be equal in order to exploit the complete capacity offered by the channel of SAC and offer both orthogonal pulses if required as well as perfect reconstruction ability of the resulting DFT FB [8].

\subsection{Spectrum Sensing and Access}

The two important phases of CR, SSE and SAC, are modeled in Fig. 1 where we assume firstly that the switch is in the SSE position. In this case, the input of the SU RX contains a thermal noise modeled as complex zero-mean AWGN with variance $\sigma_{v}^{2}$ as well as potential interference resulting by third parties including the PU signal. Thus the input signal $y[n]=v[n]$ is represented at the output of the DFT analysis FB whose filters have the $z$-transform 


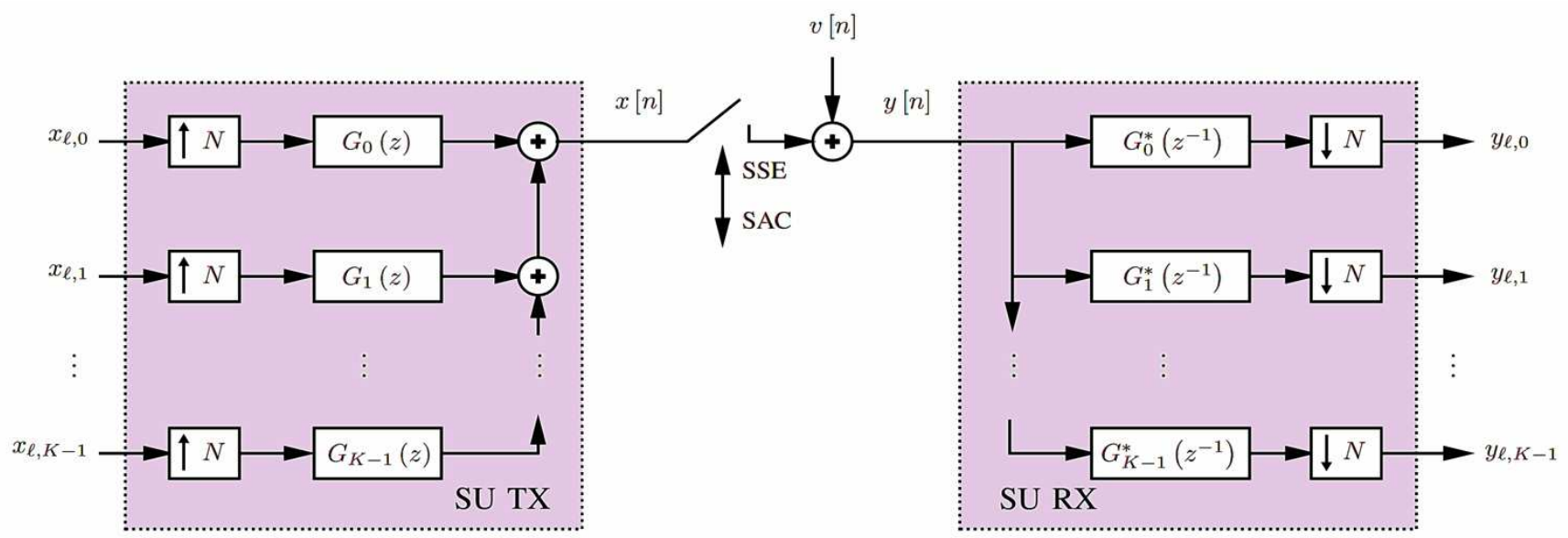

Figure 1. System model with DFT modulated FBs.

$G_{k}^{*}\left(z^{-1}\right)$ with

$$
G_{k}(z)=G\left(z W^{k}\right), \quad k=0, \ldots, K-1
$$

where $\quad W=\mathrm{e}^{-j \frac{2 \pi}{K}}$ and $\quad G(z)=\sum_{n=0}^{L-1} g[n] z^{-n} \quad$ is the $z$ - transform of $\mathbf{g}$. To this end, the signals are downsampled by a factor $N$ and projected onto the corresponding pulse to provide the TF projections $y_{\ell, k}$ assumed to be jointly Gaussian distributed where the elements of the vector $\mathbf{y}_{\ell}=\left[y_{\ell, 0}, \ldots, y_{\ell, K-1}\right]$ at the output of the analysis FB are given by

$$
\begin{aligned}
y_{\ell, k} & =\sum_{n \in \mathbb{Z}} v[n] g^{*}[n-\ell N] \mathrm{e}^{-j 2 \pi(n-\ell N) k / K} \\
& =\sum_{n \in \mathbb{Z}} v[n] g^{*}[n-\ell N] \mathrm{e}^{-j 2 \pi n k / K}
\end{aligned}
$$

To determine whether $y_{\ell, k}$ at the $k$ th frequency and $l$ th time slots is occupied for transmitting the PU signal or available for the SU to transmit its data, e.g. in interweave $\mathrm{CR}$, a threshold test is performed usually in combination with a suitable prediction. Secondly, we assume for simplicity that the aforementioned SSE has indicated availability of all subbands for SU transmission. In this case, the switch in Fig. 1 is in the SAC position and the SU TX vector $\mathbf{x}_{\ell}=\left[x_{\ell, 0}, \ldots, x_{\ell, K-1}\right]$ is upsampled by a factor $N$ and the signal $x[n]$ is synthesized by $K$ synthesis filters with $z$ - transforms $G_{k}(z)$ according to

$$
\begin{aligned}
x[n] & =\sum_{k=0}^{K-1} \sum_{\ell \in \mathbb{Z}} x_{\ell, k} g[n-\ell N] \mathrm{e}^{j 2 \pi(n-\ell N) k / K} \\
& =\sum_{k=0}^{K-1} \sum_{\ell \in \mathbb{Z}} x_{\ell, k} g[n-\ell N] \mathrm{e}^{j 2 \pi n k / K}
\end{aligned}
$$

Upon reception of $y[\ell N+L-1]$, two tasks should be executed at the SU RX, namely detecting $\mathbf{x}_{\ell}$ and simultaneously taking a decision about the presence of a PU signal in the $\ell$ th time slot [8]. Clearly, both tasks benefit from good TF concentration properties of $\mathbf{g}$ that will be discussed in Sect. 5.

\subsection{Self-Interference}

If the functions $\left\{g_{\ell, k}\right\}$ are not orthogonal in both time and frequency domains, the demodulation of $\mathbf{y}_{\ell}$ representing sufficient statistics for the detection of $\mathbf{x}_{\ell}$ in AWGN is subject to self-interference. We define $\hat{x}_{\ell, k}=y_{\ell, k} /\|\mathbf{g}\|^{2}$ as the estimated value of $x_{\ell, k}$ given by

$$
\hat{x}_{\ell, k}=x_{\ell, k}+\rho_{\Omega_{\mathrm{lBI}}^{(\ell, K)}}+\rho_{\Omega_{\mathrm{CBI}}^{(\ell, K)}}+\rho_{\Omega_{\mathrm{RI}}^{(\ell, K)}}+z_{\ell, k}
$$

where

$$
\rho_{\Omega}=\sum_{(\lambda, \kappa) \in \Omega} x_{\lambda, \kappa} \rho_{\ell-\lambda, k-\kappa}
$$

Here,

$$
\rho_{\lambda, \kappa}=\sum_{n=0}^{L-1} g[n] g^{*}[n-\lambda N]\|\mathbf{g}\|^{-2} \mathrm{e}^{-j 2 \pi n \kappa / K} \quad \text { denotes }
$$
crosscorrelation and $\rho_{\Omega_{\mathrm{IBI}}^{(\ell, K)}}, \rho_{\Omega_{\mathrm{CBI}}^{(\ell, K)}}$ and $\rho_{\Omega_{\mathrm{RI}}^{(\ell, K)}}$ are defined as the intra-band interference (IBI), the cross-band interference (CBI) and the residual interference (RI), respectively. Moreover, $z_{\ell, k}=\left\langle v[n], g_{\ell, k}[n]\right\rangle|| \mathbf{g} \|^{-2}$ defines the AWGN noise and the third party interference contributions in $\hat{x}_{\ell, k}$ with variance equal to $\mathbf{E}\left\{\left|z_{\ell, k}\right|^{2}\right\}=\left.\sigma_{v}^{2}|| \mathbf{g}\right|^{-2}=\sigma_{z}^{2}$. In (1), we define the sets $\Omega_{\mathrm{IBI}}^{(\ell, k)}, \Omega_{\mathrm{CBI}}^{(\ell, k)}$ and $\Omega_{\mathrm{RI}}^{(\ell, k)}$ as

$$
\begin{aligned}
& \Omega_{\mathrm{IBI}}^{(\ell, k)}=\left\{(\lambda, \kappa): \lambda \in \Omega_{\mathrm{T}, \ell}, \kappa=k\right\} \\
& \Omega_{\mathrm{CBI}}^{(\ell, k)}=\left\{(\lambda, \kappa): \lambda=\ell, \kappa \in \Omega_{\mathrm{F}, \mathrm{k}}\right\}
\end{aligned}
$$




$$
\Omega_{\mathrm{RI}}^{(\ell, k)}=\left\{(\lambda, \kappa): \lambda \in \Omega_{\mathrm{T}, \ell}, \kappa \in \Omega_{\mathrm{F}, \mathrm{k}}\right\}
$$

with index sets in frequency and time given by

$$
\begin{gathered}
\Omega_{\mathrm{F}, k}=\{0, \ldots, K-1\} /\{k\} \\
\Omega_{\mathrm{T}, \ell}=\{\ell-\Delta, \ldots, \ell-1, \ell+1, \ldots, \ell+\Delta\} \\
\Delta=\left\lceil\frac{L}{N}\right\rceil-1 .
\end{gathered}
$$

By exploiting the central limit theorem, the IBI, CBI and RI contributions are modeled as zero-mean Gaussian random variables with variance [8]

$$
\sigma_{\rho}^{2}=\mathbf{E}\left\{\left|\rho_{\Omega_{\mathrm{IBI}}^{(\ell, k)}}+\rho_{\Omega_{\mathrm{CBI}}^{(\ell, k)}}+\rho_{\Omega_{\mathrm{RI}}^{(\ell, k)}}\right|^{2}\right\}=\sum_{(\lambda, \kappa) \in \Omega_{\mathrm{IBI}}^{(\ell, k)} \cup \Omega_{\mathrm{CBI}}^{(\ell, k)} \cup \Omega_{\mathrm{RI}}^{(\ell, k)}}\left|\rho_{\ell-\lambda, k-K}\right|^{2}
$$

Here, we consider $x_{\ell, k}$ to be mutually independent DQPSK symbols with unit bit energy. Fig. 2 illustrates the self-interference affecting on demodulating the signal $x_{0,3}$ and the corresponding sets for $\hat{x}_{0,3}[8]$.

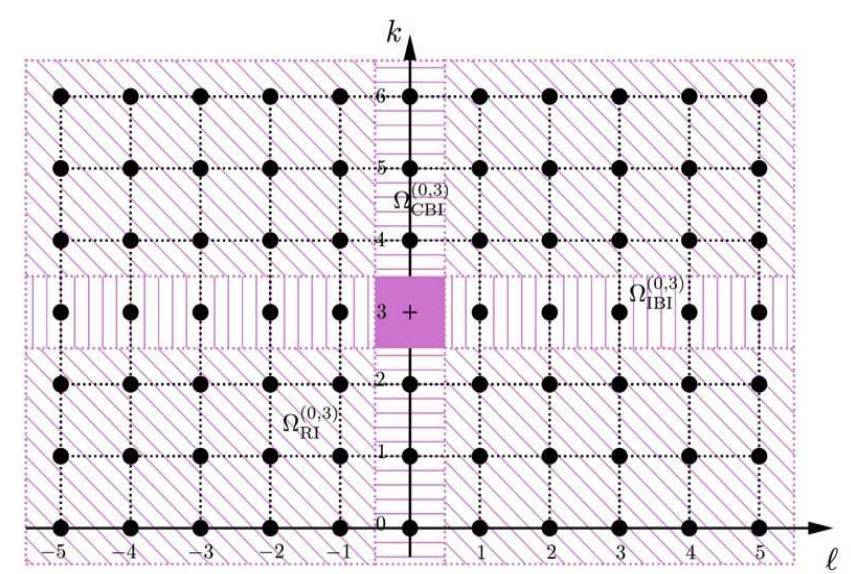

Figure 2. Self-interference to $x_{0,3}$ with $K=7$ and $\Delta=5: I B I, C B I$ and $R I$ hatched vertical, horizontal and diagonal, respectively.

\section{Transceiver Design}

\subsection{Optimization Constraints}

The main task in designing the transceiver in Fig. 1 is optimizing $\mathbf{g}$ subject to certain constraints. Here, two types of constraints are distinguished.

Firstly, we consider the constraints arising from system specifications formulated as a lower bound $R_{\min }$ on the data transmission rate $R$ in bit/s and an upper bound $\bar{P}_{\mathrm{b}, \max }$ on the average BER $\bar{P}_{\mathrm{b}}$ of the $\mathrm{SU}$ in form of

$$
\begin{aligned}
& R \geq R_{\text {min }} \\
& \bar{P}_{\mathrm{b}} \leq \bar{P}_{\mathrm{b}, \max } .
\end{aligned}
$$

The constraint in (2) is met upon choosing an appropriate value $K$ as explained in Sect. 4 independently of the form of g. On the contrary, the constraint (3) depends on $\mathbf{g}$ and has therefore to be taken into account in the aforementioned optimization. In view of the Gaussian interference approximation in Sect. 2.3, the average BER reads [9]

$$
\bar{P}_{\mathrm{b}}(\zeta)=\mathrm{Q}_{1}\left(a_{-}, a_{+}\right)-\frac{1}{2} I_{0}\left(a_{-} a_{+}\right) \exp ^{\left[-\frac{1}{2}\left(a_{-}^{2}+a_{+}^{2}\right)\right]}
$$

with $a_{ \pm}=a_{ \pm}(\zeta)=\sqrt{(2 \pm \sqrt{2}) \zeta}$ and $\zeta=\frac{1}{\sigma_{v}^{2}+\sigma_{\rho}^{2}}$. Furthermore, $I_{0}(x)$ and $\mathrm{Q}_{1}(a, b)$ are the zeroth order modified Bessel function and the Marcum Q function [9], respectively. For a given value $\bar{P}_{\mathrm{b}, \max }$ in (3), the constraint can be formulated as

$$
\mathrm{C}_{1} \quad: \zeta \geq \bar{P}_{\mathrm{b}}^{-1}\left(\bar{P}_{\mathrm{b}, \max }\right)
$$

The second type of constraints arises from implementation aspects. The norm of $\mathbf{g}$ is limited to one, i.e. we have

$$
\mathrm{C}_{2} \quad:\|\mathbf{g}\|=1 .
$$

Finally, in order to obtain a $\mathbf{g}$ providing a system $\left\{g_{\ell, k}[n]\right\}$ of pulses being orthogonal in both time and frequency, a necessary condition reads

$$
\mathrm{C}_{3}: g[n]=g[L-n-1] \text { for } n \in\{0, \ldots, L-1\}
$$

in conjunction with $\mathbf{g} \in \mathbb{R}^{L} \quad$ [10]. For later use, we collect the three constraints in (5), (6) and (7) symbolically in the vector

$$
\text { C: }\left[\mathrm{C}_{1}, \mathrm{C}_{2}, \mathrm{C}_{3}\right] .
$$

\subsection{Objective Functions}

Our objective is the minimization of a dispersion metric of the pulse $\mathbf{g}$ subject to the constraints $\mathbf{C}$. Four different metrics are considered below.

\subsubsection{Heuristic Dispersion Measure}

A heuristic dispersion measure (HDM) is considered as the first approach [8]. Here, the time index $n$ in $\mathbf{g}$ is interpreted as a random variable whose probability $\operatorname{Pr}(\theta=n)$ is defined by $\operatorname{Pr}(\theta=n)=g[n]^{2} /\|\mathbf{g}\|^{2}$. In view of (6), we have $\operatorname{Pr}(\theta=n)=g[n]^{2}$ and choose the variance of $\theta$ as the dispersion metric, i.e.

$$
\begin{aligned}
\eta_{\mathrm{HDM}}(\mathbf{g}) & =\sum_{n=0}^{L-1} n^{2} \operatorname{Pr}(\theta=n)-\left[\sum_{n=0}^{L-1} n \operatorname{Pr}(\theta=n)\right]^{2} \\
& =\mathbf{g}^{\mathrm{T}} \mathbf{D g}-\left(\mathbf{g}^{\mathrm{T}} \mathbf{Z g}\right)^{2}
\end{aligned}
$$


with $\mathbf{D}=\operatorname{Diag}\left[0, \ldots,(L-1)^{2}\right]$ and $\mathbf{Z}=\operatorname{Diag}[0, \ldots, L-1]$. Thus the optimization problem based on the heuristic dispersion measure reads

$$
\mathbf{g}_{\mathrm{HDM}}=\underset{g \in L^{2}(\mathbb{Z})}{\arg \min } \eta_{\mathrm{HDM}}(\mathbf{g}) \quad \text { s.t. } \mathbf{C} \text {. }
$$

\subsubsection{Time-Frequency Concentration}

In order to obtain a pulse with TF concentration (TFC), firstly, we measure the energy of the pulse $\psi_{\Omega_{\Delta,,}, \Omega_{\Delta, F}}(\mathbf{g})$ inside a given TF window $\Omega_{\Delta, \mathrm{T}} \times \Omega_{\Delta, \mathrm{F}}$ based on the Rihaczek distribution [6]. Secondly, the leakage of the pulse energy outside the TF window is to be minimized. Here, we choose

$$
\Omega_{\Delta, \mathrm{T}} \times \Omega_{\Delta, \mathrm{F}}=\left\{\frac{L-\Delta_{\mathrm{T}}}{2}+1, \ldots, \frac{L+\Delta_{\mathrm{T}}}{2}\right\} \times\left[-\frac{\Delta_{\mathrm{F}}}{2}, \frac{\Delta_{\mathrm{F}}}{2}\right),
$$

where $L+\Delta_{\mathrm{T}}$ is assumed even, $\Delta_{\mathrm{T}}$ is a positive integer chosen as $\Delta_{\mathrm{T}}=N$ and $\Delta_{\mathrm{F}}=1 / K$. The objective function now becomes

$$
\eta_{\mathrm{TFC}}(\mathbf{g})=1-\frac{\psi_{\Omega_{\Delta, r}, \Omega_{\Delta, F}}(\mathbf{g})}{\|\mathbf{g}\|^{2}}=1-\psi_{\Omega_{A, T}, \Omega_{\Delta, F}}(\mathbf{g}),
$$

where we define [6]

$$
\psi_{\Omega_{\Delta, T}, \Omega_{\Delta, \mathrm{F}}}(\mathbf{g})=\sum_{n \in \Omega_{\Delta, \mathrm{T}}} \int_{f \in \Omega_{\triangle, \mathrm{F}}} R_{\mathbf{g}}(n, f) \mathrm{d} f
$$

with $\mathrm{R}_{g}(n, f)=g[n] \sum_{v \in \mathbb{Z}} g[v] \mathrm{e}^{j 2 \pi f(v-n)}$ known as the Rihaczek distribution [11]. The energy of the pulse can be formulated in a matrix form as $\psi_{\Omega_{\Delta, T}, \Omega_{\Delta, \mathrm{F}}}(\mathbf{g})=\mathbf{g}^{\mathrm{T}} \mathbf{S} \overline{\mathbf{g}}$ where $\quad S=\left\{S_{n, v}\right\}=\left\{\Delta_{\mathrm{F}} \operatorname{sinc}\left(\Delta_{\mathrm{F}} \pi(n-v)\right)\right\} \quad$ is $\quad$ a $(L \times L)$-dimensional matrix and the reduced pulse $\overline{\mathbf{g}}$ is formulated as

$$
\overline{\mathbf{g}}=\left\{0, \ldots, 0, g\left[\frac{L-\Delta_{\mathrm{T}}}{2}+1\right], \ldots, g\left[\frac{L+\Delta_{\mathrm{T}}}{2}\right], 0, \ldots, 0\right\}^{\mathrm{T}} .
$$

Therefore, the optimization problem based on pulse energy in TF domain is given by

$$
\mathbf{g}_{\mathrm{TFC}}=\underset{g \in L^{2}(\mathbb{Z})}{\arg \min } \eta_{\text {TFC }}(\mathbf{g}) \quad \text { s.t. } \mathbf{C} .
$$

where $\eta_{\mathrm{TFC}}(\mathbf{g})=1-\mathbf{g}^{\mathrm{T}} \mathbf{S} \mathbf{g}$.

\subsubsection{Time Concentration}

In a corresponding time concentration (TC) approach, the concentration is only considered in time while the frequency window $\Omega_{\Delta, \mathrm{F}}$ is extended to the interval $\Omega_{\mathrm{F}}=[0,1)[8]$. Consequently, the optimization function for TC reads

$$
\eta_{\mathrm{TC}}(\mathbf{g})=1-\frac{\psi_{\Omega_{\Delta, T}, \Omega_{\mathrm{F}}}(\mathbf{g})}{\|\mathbf{g}\|^{2}}=1-\psi_{\Omega_{\Delta, T}, \Omega_{\mathrm{F}}}(\mathbf{g})
$$

with $\psi_{\Omega_{\Delta, T}, \Omega_{\mathrm{F}}}(\mathbf{g})=\sum_{n \in \Omega_{\Delta, \mathrm{T}}} \int_{\in \Omega_{\mathrm{F}}} R_{\mathbf{g}}(n, f) \mathrm{d} f=\sum_{n \in \Omega_{\Delta, \mathrm{T}}} g[n]^{2}$.

The optimized problem is formulated as

$$
\mathbf{g}_{\mathrm{TC}}=\underset{g \in L^{2}(\mathbb{Z})}{\arg \min } \eta_{\mathrm{TC}}(\mathbf{g}) \quad \text { s.t. } \mathbf{C} .
$$

where $\eta_{\mathrm{TC}}(\mathbf{g})=1-\|\overline{\mathbf{g}}\|^{2}$.

\subsubsection{Frequency Concentration}

To formulate the frequency concentration (FC) metric, we extend the time window interval $\Omega_{\Delta, \mathrm{T}}$ to $\Omega_{\mathrm{T}}=\{0, \ldots, L-1\}$. The corresponding optimized problem becomes

$$
\mathbf{g}_{\mathrm{FC}}=\underset{g \in L^{2}(\mathbb{Z})}{\arg \min } \eta_{\mathrm{FC}}(\mathbf{g}) \quad \text { s.t. } \mathbf{C} .
$$

with

$$
\eta_{\mathrm{FC}}(\mathbf{g})=1-\frac{\psi_{\Omega_{\mathrm{T}}, \Omega_{\Delta F}}(\mathbf{g})}{\|\mathbf{g}\|^{2}}=1-\psi_{\Omega_{\mathrm{T}}, \Omega_{\Delta \mathrm{F}}}(\mathbf{g})=1-\mathbf{g}^{\mathrm{T}} \mathbf{S g} .
$$

\section{Practical Implementation Based on the WLAN Standard IEEE802.11g}

In this section we use the aforementioned DFT modulated FB transceiver designs to consider practical CR implementations with physical layer parameters resembling IEEE $802.11 \mathrm{~g}$. That is, we define the system parameters including the modulation scheme such that the required data transmission rate of IEEE $802.11 \mathrm{~g}$ can be reached. For complexity reasons, DQPSK is used to avoid the need for channel estimation in differential detection of the received data symbols. In IEEE $802.11 \mathrm{~g}$, the minimum data rate in (2) to be supported by the CR system is $R_{\min }=54 \mathrm{Mbit} / \mathrm{s}$. We assume a sampling rate $\alpha=30 \mathrm{Msample} / \mathrm{s}$ and choose $K=512$. Clearly, in view of the 2-bits carried by one DQPSK symbol, the maximum data rate $R$ results to 60 $\mathrm{Mbit} / \mathrm{s}$. For the aforementioned parameters, we can thus leave subcarriers at the frequency band boundaries unloaded in order to have a certain separation to neighboring bands. More accurately, we use the 462 subcarriers in the band center and leave 25 subcarriers at both band edges unloaded as shown in Fig. 3. The resulting duration of a symbol carrying 512 DQPSK symbols is about $17 \mu \mathrm{s}$.

Furthermore, we choose $L=1536=\bar{L} K$ as the length of our optimized pulse $\mathbf{g}$ with $\bar{L}=3$. Note that the TF concentration of $\mathbf{g}$ being important for the SSE 
performance depends mainly on the TF resolution parameters $\Delta_{\mathrm{T}}=512$ and $\Delta_{\mathrm{F}}=1 / 512$. Concerning possible approaches for solving the optimization problems in Sect. 3, one has to realize that these problems are highly nonlinear. Thus, using interior-point (IP) and sequential quadratic programming (SQP) are powerful methods which can effectively solve our optimization problems easily and very reliably with a small number of iterations required for convergence [12], [13].

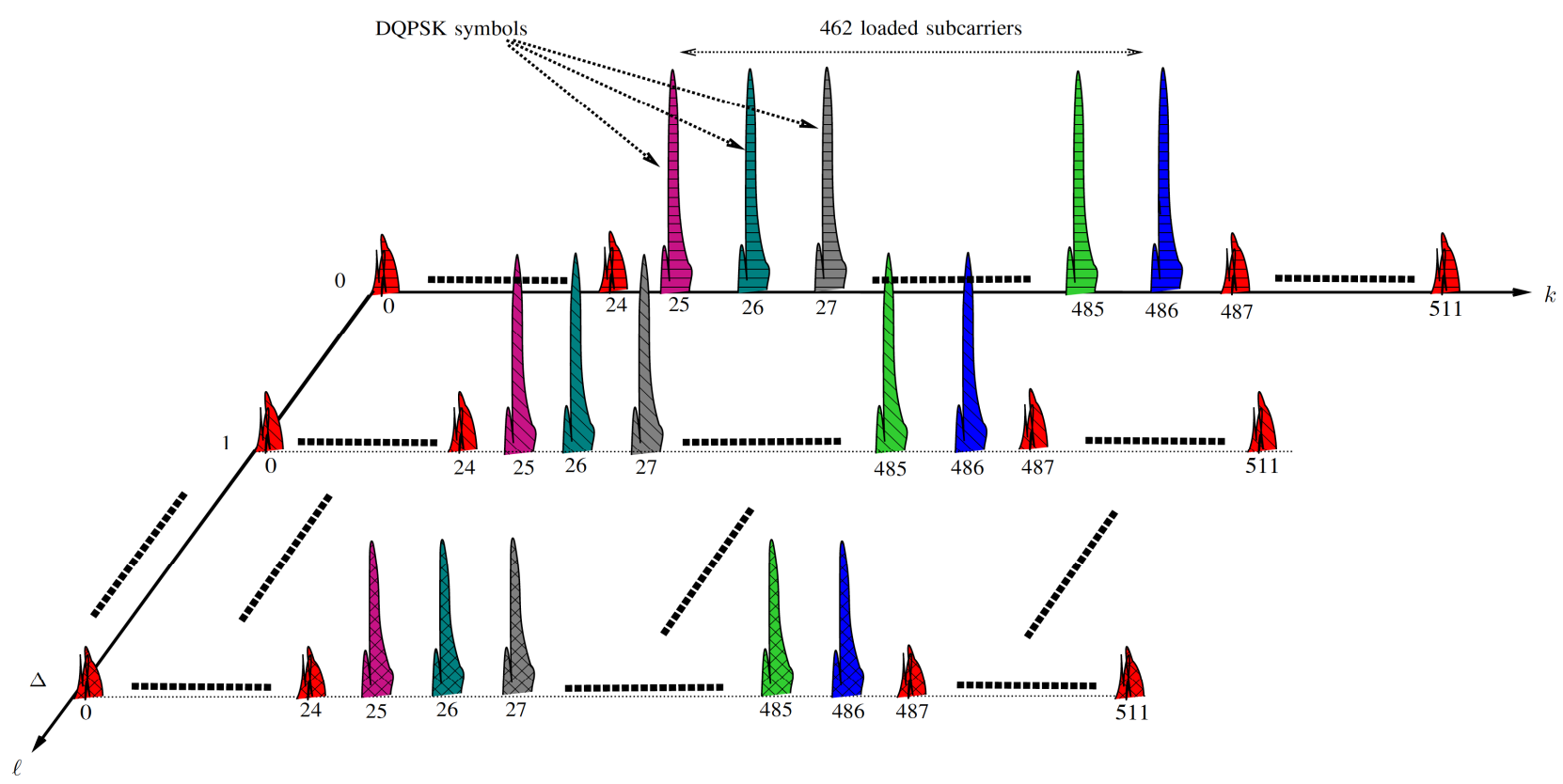

Figure 3. The loaded and unloaded subcarriers for reaching the data transmission rate of IEEE $802.11 \mathrm{~g}$.

Due to the large value of $K$, a polyphase structure for implementing the FBs at both transmitter and receiver provides a considerable saving in complexity [10]. To be more specific, we choose the $k$ th analysis polyphase subfilter as $\mathbf{e}_{k}=\left[e_{k}[0], \ldots, e_{k}[\bar{L}-1]\right]^{\mathrm{T}}$ being obtained from $\mathbf{g}$ by an $N$-fold downsampling with phase shift $k$, i.e.

$$
e_{k}[n]=g[N n+k] \text {. }
$$

If the transmitter and the receiver use a common hardware architecture, we can make use of the fact that the $k$ th synthesis polyphase subfilter $r_{k}[n]$ satisfies $r_{k}[n]=e_{K-1-k}[n]$, so that the synthesis filter is the permutation of the corresponding analysis filter which leads to corresponding saving in filter implementation. Note that each of the polyphase subfilters has a number of coefficients $\bar{L}=3$ which is a factor of $K$ less than the number of coefficients in the original pulse $\mathbf{g}$.

Now we compare the computational complexity $C$ measured in units of filter operations per second (FOPS) [14] required using the original FB in Fig. 1 with the complexity $\bar{C}$ due to applying the aforementioned polyphase structure. In the original FB implementation, the complex input signal is sampled at a rate $\alpha$ and three real multipliers are required to perform the multiplication of two complex numbers. Thus we have for the filtering

$$
C=3 \alpha K L=3 \cdot 30 \cdot 10^{6} \cdot 512 \cdot 1536 \text { FOPS } \approx 71 \cdot 10^{12} \mathrm{FOPS} .
$$

By applying the polyphase structure, two real multipliers are required to perform the multiplication of the complex input signal and the real-valued coefficients of $e_{k}[n]$. Furthermore, the polyphase subfilters are computed at a sampling rate $\frac{\alpha}{N}$. Thus,

$$
\bar{C}=\frac{\alpha}{N}\left(2 K \bar{L}+\frac{K}{2} \operatorname{ld} K\right) \approx 315 \cdot 10^{6} \mathrm{FOPS},
$$

where $\frac{K}{2} \operatorname{ld} K$ is the DFT implementation complexity. The complexity reduction is thus $\frac{C}{\bar{C}}=225 \cdot 10^{3}$.

\section{Performance Analysis}

The performance analysis is based on two parts. First, the energy concentration of the pulses governing the SSE performance is considered. Secondly, we study the SAC performance in terms of the achievable BER for a given pulse resulting from the constrained optimization in Sect. 3. Before starting the analysis, we introduce standard parameters, namely the signal-to-interference-plus-noise ratio (SINR) 
$\zeta=\frac{1}{\sigma_{v}^{2}+\sigma_{\rho}^{2}} \quad, \quad$ the $\quad$ signal-to-noise ratio (SNR) $\gamma=\frac{1}{\sigma_{v}^{2}}=\frac{1}{\sigma_{z}^{2}}$, the signal-to-interference ratio (SIR) $\xi=\frac{1}{\sigma_{\rho}^{2}}$ and finally the loss due to possible self-interference and PU interference $\delta=\frac{\gamma}{\zeta}=1+\frac{\sigma_{\rho}^{2}}{\sigma_{v}^{2}}$. Note that subsequently, a certain value $\delta$ is assumed given for both the optimization and a corresponding simulation scenario.

The TF concentration is characterized in terms of the leakage of the optimized pulses as a function of $\delta \in[0,3 \mathrm{~dB}]$ and $\gamma$, where $\delta=0 \mathrm{~dB}$ represents a situation with zero self-interference, i.e. $\sigma_{\rho}^{2}=0$. For $\gamma=8 \mathrm{~dB}$, the resulting pulses are shown in Fig. 4 for the four different optimization approaches in Sect. 3. As can be seen, in time domain and for $\delta=0 \mathrm{~dB}$, both HDM and TC based pulses take shapes being close to a rectangular window and show better concentration within $\Delta_{\mathrm{T}}$ than TFC and FC based g. A coarse inspection of Fig. 4 suggests furthermore that for increasing $\delta$, the pulse concentration decreases and the shapes differ according to the type and quantity of the interference term. To judge the TF concentration in greater detail, the absolute value of the frequency-discrete Rihaczek distribution $\left|\mathrm{R}_{g, \mathrm{~d}}(n, f)\right|=\left|\int_{\frac{k}{K}-\frac{\Delta_{\mathrm{F}}}{2}}^{\frac{k}{K}+\frac{\Delta_{\mathrm{F}}}{2}} \mathrm{R}_{g, \mathrm{~d}}(n, f) \mathrm{df}\right|$ in $[\mathrm{dB}]$ is illustrated in Fig. 5 for $\delta=3 \mathrm{~dB}$ and $\gamma=8 \mathrm{~dB}$. For the HDM and TC cases, the latter shows the best concentration in time domain and both of them have almost zero IBI and RI, but non-zero ICI due to the aforementioned allowed loss. Correspondingly, the FC case experiences zero ICI while IBI and RI are about the same as for the TFC case. Next, we consider the leakage values $\eta_{\mathrm{TC}}(),. \eta_{\mathrm{FC}}($.$) and \eta_{\mathrm{TFC}}($.$) for \mathbf{g}_{\mathrm{HDM}}, \mathbf{g}_{\mathrm{TFC}}, \mathbf{g}_{\mathrm{TC}}$ and $\mathbf{g}_{\mathrm{FC}}$ as a function of $\xi=\gamma$,i.e. $\delta=3 \mathrm{~dB}$, in Fig. 6. Clearly, all leakage measures decrease for increasing values of $\xi$ due to a decreasing interference term. Apparently, as a direct consequence of the large value of $K$, the leakage $\eta_{\mathrm{FC}}($.$) of$ any pulse is very close to zero and according to the pulse design, the leakage $\eta_{\mathrm{TFC}}($.$) is the largest among all measured$ leakages for all pulses. Obviously, the objective function affects the leakage measure as can be seen from $\eta_{\mathrm{TC}}\left(\mathbf{g}_{\mathrm{TC}}\right), \eta_{\mathrm{TC}}\left(\mathbf{g}_{\mathrm{HDM}}\right)$, and $\eta_{\mathrm{FC}}\left(\mathbf{g}_{\mathrm{FC}}\right)$ for which the corresponding leakages are zero. The relevant leakage measure for SSE in the TF domain is clearly $\eta_{\mathrm{TFC}}($.$) . Here,$ the least leakage metric is achieved by $\eta_{\mathrm{TFC}}\left(\mathbf{g}_{\mathrm{TFC}}\right)$ which is almost identical to $\eta_{\mathrm{FC}}\left(\mathbf{g}_{\mathrm{FC}}\right)$.

The BER performance is considered for two cases in Fig. 7, namely interference-free transmission with bi-orthogonal pulses and thus $\delta=0 \mathrm{~dB}$ as well as transmission with interference and non-orthogonal pulses where again $\delta=3 \mathrm{~dB}$. In the first case, it is seen that both $\bar{P}_{\mathrm{b}}$ resulting from optimization (opt) and simulations (sim) are identical to the theoretical expressions for DQPSK in AWGN (4). However, for $\delta=3 \mathrm{~dB}$, the self-interference is not Gaussian anymore so that a deviation of the simulated BERs from the ones in the optimization constraints arises where the latter are higher than the former. This in turn can be taken into account in the optimization procedure as long as the deviation is less than $\delta$. For example, if we want to design a system based on the TFC optimization criterion with $\delta=3 \mathrm{~dB}$ maximum allowable interference and $\gamma=12 \mathrm{~dB}$ where the deviation between the both curves in Fig. 7 can be observed to be $1.7 \mathrm{~dB}$, a value of $\zeta=12 \mathrm{~dB}-1.3 \mathrm{~dB}=10.7 \mathrm{~dB}$ should be employed in the optimization to satisfy the BER constraint.

\section{Conclusions}

Trading implementation complexity in a cognitive radio transceiver against high-resolution spectrum sensing and minimum bit-error rate performance in spectrum access can be taken into account in the design of suitable DFT modulated filter banks. The system specifications translate into a constrained optimization procedure for finding corresponding prototype filter coefficients. If the design is applied to a system with characteristics similar to IEEE $802.11 \mathrm{~g}$, the system specifications can be met by properly treating interference phenomena arising from both third parties as well as from self-interference in the filter bank. The transceiver complexity can benefit from the polyphase implementation of DFT modulated filter banks.

The approach is currently extended to the case of time-/frequency selective fading channels [16] where the channel parameterization translates into properties of the corresponding TF signal representations, and the resulting pulses will be applied to indoor cognitive radio environments.

\section{Acknowledgments}

This work has been supported by the German Federal Ministry of Education and Research project Entwurf einer Cognitive Radio-ARchitektur basierend auf Optimierten Zeit-FreqUenz-SignALdarstellungen (CAROUSAL). 

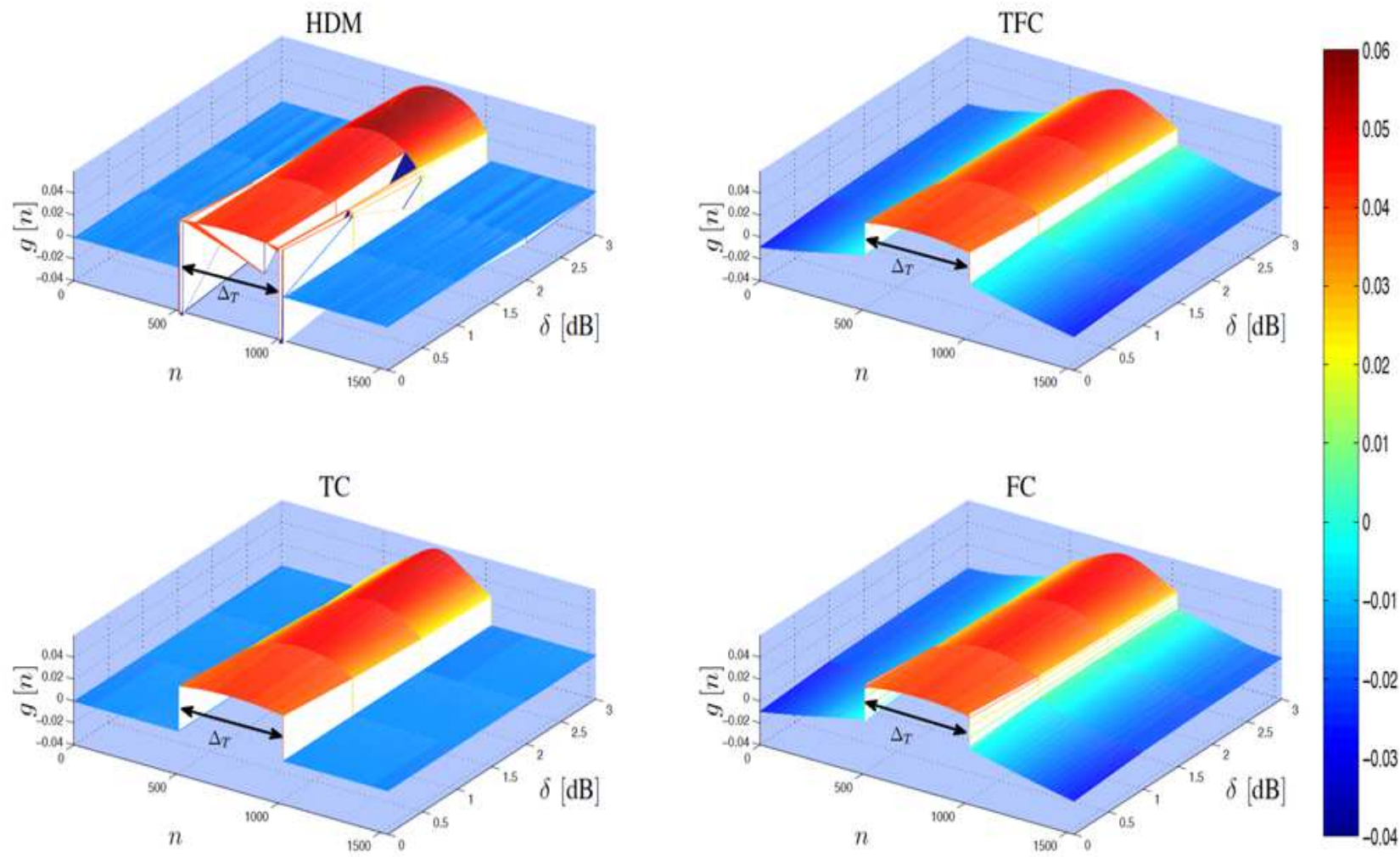

Figure 4. The optimized pulses in case of different values of $\delta$ using HDM, TFC, TC and FC and $\gamma=8 \mathrm{~dB}$.
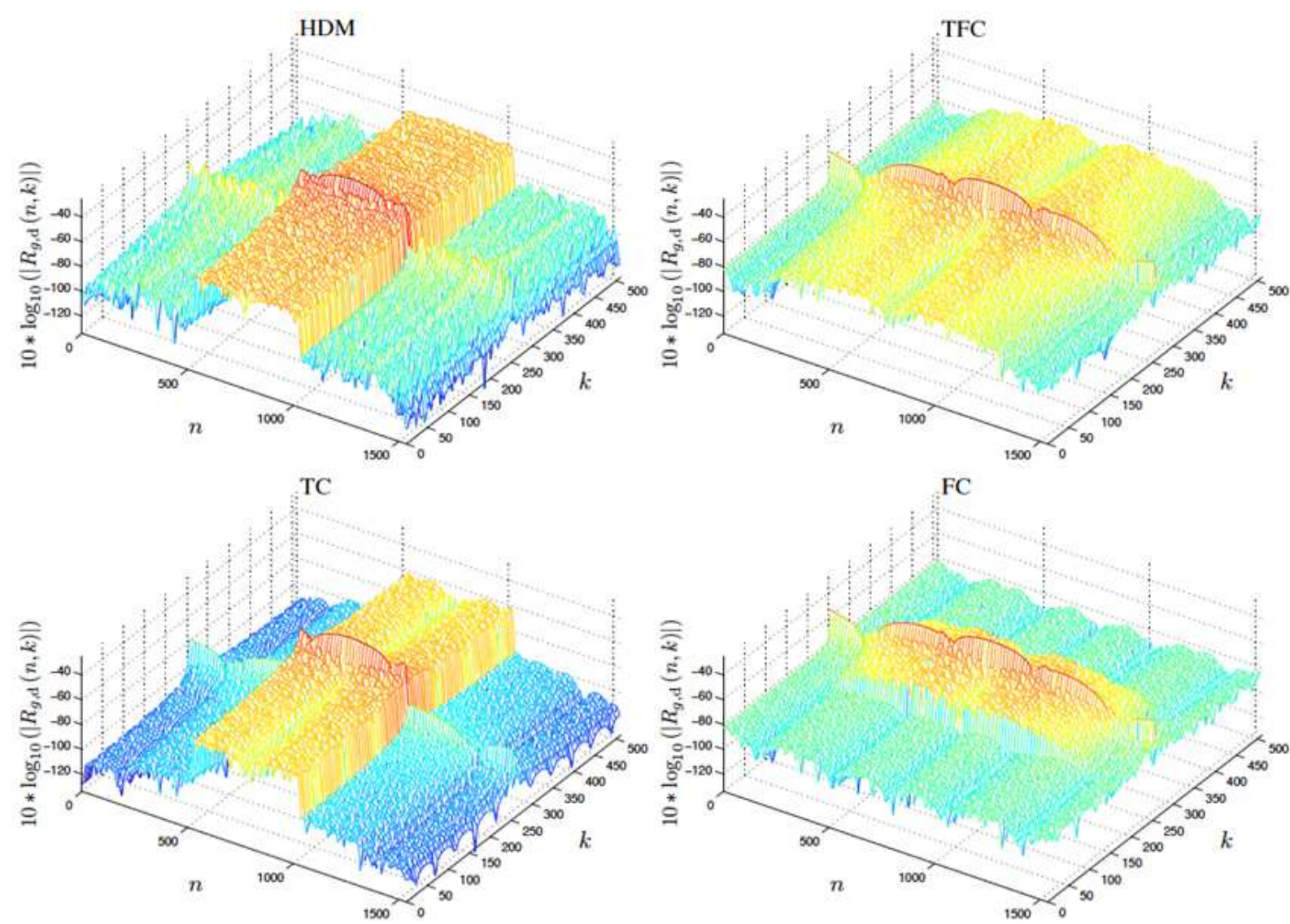

FC

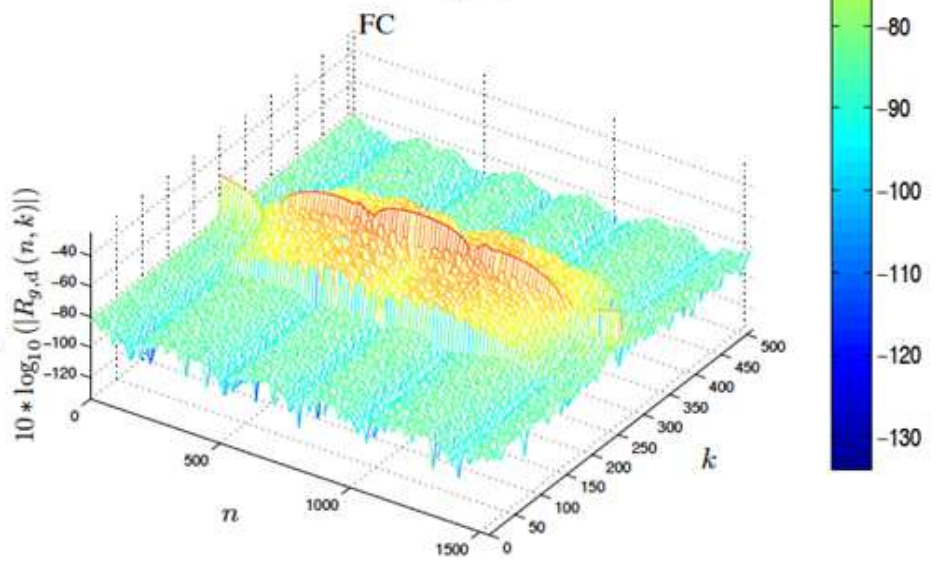

Figure 5. Energy concentration in TF plane based on frequency-discrete Rihaczek distribution $R_{\mathrm{g}, \mathrm{d}}(n, k)$ using HDM, TFC, TC and FC for $\delta=3 \mathrm{~dB}$ and $\gamma=8 \mathrm{~dB}$. 


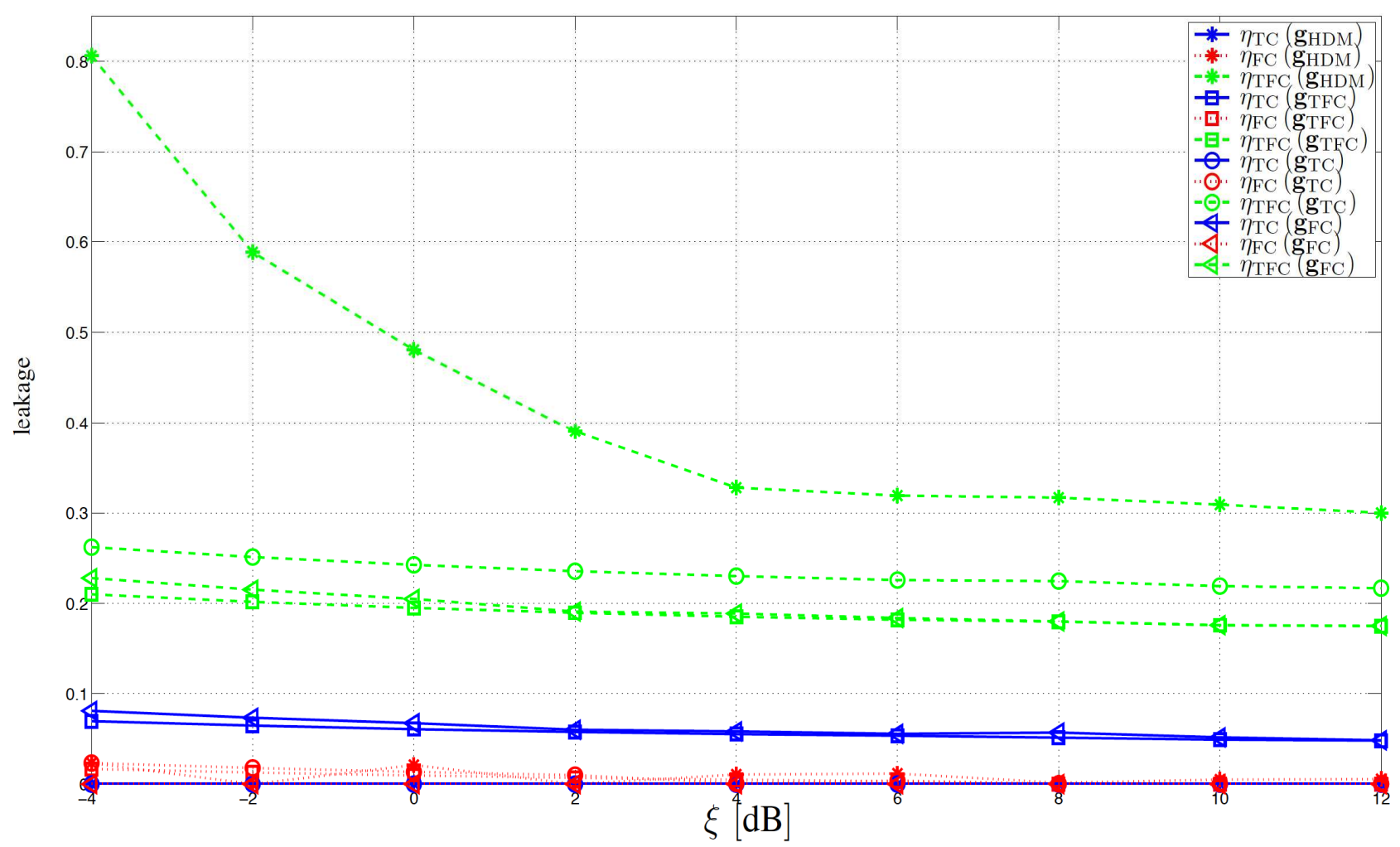

Figure 6. Leakage values of $\eta_{\mathrm{TC}}(),. \eta_{\mathrm{FC}}($.$) and \eta_{\mathrm{TFC}}($.$) for \mathbf{g}_{\mathrm{HDM}}, \mathbf{g}_{\mathrm{TFC}}, \mathbf{g}_{\mathrm{TC}}$ and $\mathbf{g}_{\mathrm{FC}}$ as a function of $\xi=\gamma$ and $\delta=3 \mathrm{~dB}$.
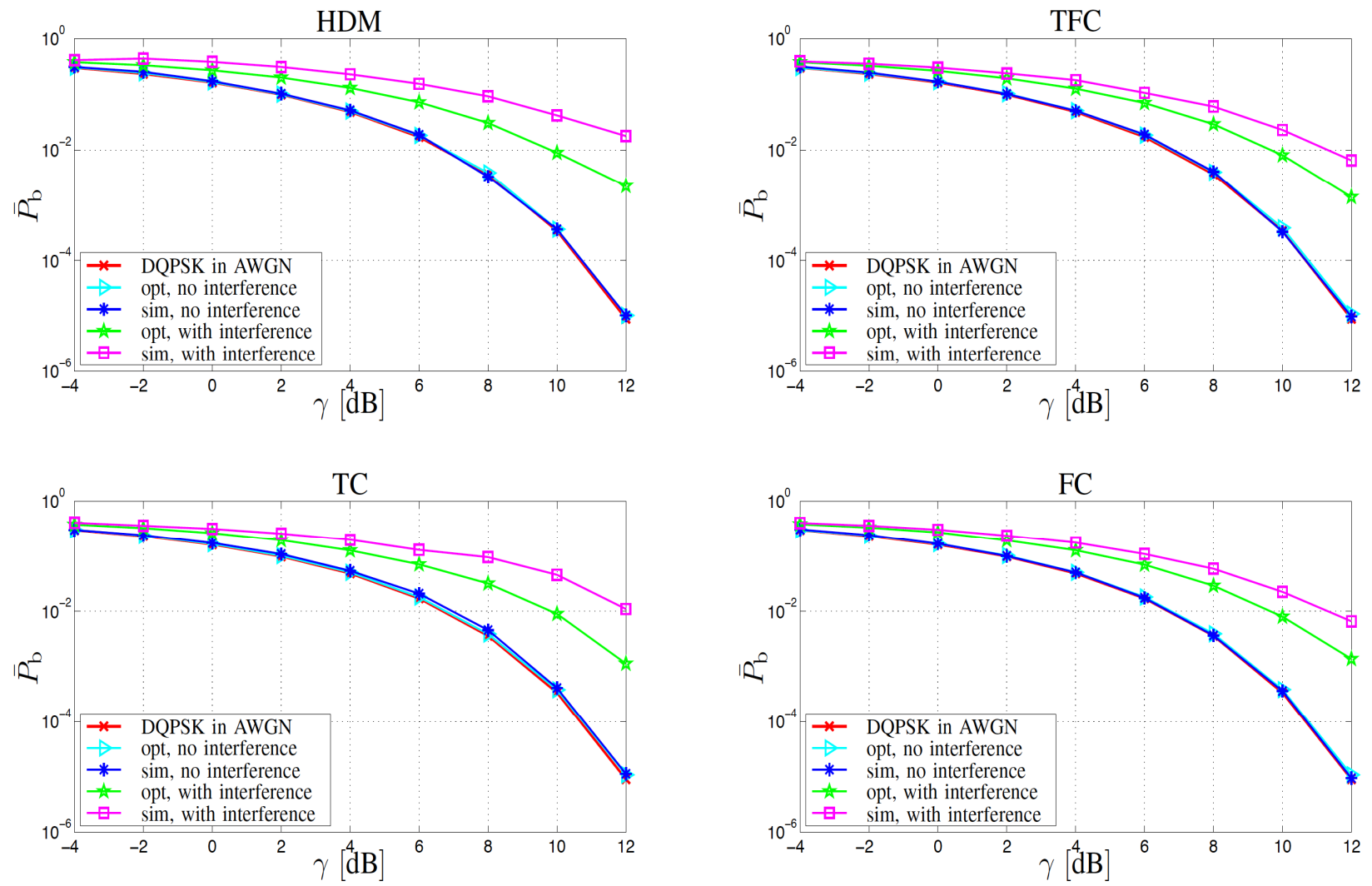

Figure 7. BER performance in $A W G N$ with DQPSK signaling in case of $\delta=0 \mathrm{~dB}$ (no interference) and $\delta=3 \mathrm{~dB}$ (with interference). 


\section{References}

[1] E. Biglieri, A. J. Goldsmith, L. J. Greenstein, N. B. Mandayam, and H. V. Poor, Principles of cognitive radio. Cambridge University press: Cambridge University Press, New York, 2013.

[2] W.-B. Chien, C.-K. Yang, and Y.-H. Huang, "Energy-saving cooperative spectrum sensing processor for cognitive radio system," circuits and systems I: Regular Papers, IEEE Transactions on, vol. 58, no. 4, pp. 711-723, April 2011.

[3] D. Joshi, D. Popescu, and O. Dobre, "Adaptive spectrum sensing with noise variance estimation for dynamic cognitive radio systems," in Information Sciences and Systems (CISS), 2010 44th Annual Conference on, March 2010, pp. 1-5.

[4] H. Bölcskei, F. Hlawatsch, and H. G. Feichtinger, "Equivalence of DFT filter banks and Gabor expansions," in Proc. of SPIE: wavelet applications in signal and image processing III, vol. 2569, July 1995, pp. 128-139.

[5] H. Feichtinger and T. Strohmer, Gabor analysis and algorithms: theory and applications, ser. Applied and numerical harmonic analysis. Birkhäuser Verlag GmbH, 2012.

[6] T. Hunziker, U. Rehman, and D. Dahlhaus, "Spectrum sensing in cognitive radios: Design of DFT filter banks achieving maximal time frequency resolution," in 8th International Conference on Information, Communications and Signal Processing (ICICS) 2011, Dec 2011, pp. 1-5.

[7] Z. Ju, T. Hunziker, and D. Dahlhaus, "Optimized paraunitary filter banks for time-frequency channel diagonalization," in EURASIP journal on advances in signal processing, vol. 2010, Dec. 2010.

[8] N. Mansour and D. Dahlhaus, "Interference in DFT modulated filter bank transceivers for cognitive radio," in European Wireless 2014; 20th European Wireless Conference; Proceedings of, May 2014, pp. 1-7.

[9] J. Proakis, Digital communications. McGraw Hill, New York, $4^{\text {th }}$ ed., 2000.

[10] P.P. Vaidyanathan, Multirate systems and filter banks. Prentice Hall Signal Processing Series, New Jersey, 1993.

[11] B. Boashash, "Time-frequency signal analysis and processing," Queensland university, Brisbane, Australia.

[12] S. Boyd and L. Vandenberghe, Convex optimization. Cambridge UK: Cambridge University Press, New York, 2004.

[13] P. Gill, W. Murray, and M. Saunders, "SNOPT: An SQP algorithm for large-scale constrained optimization," SIAM Journal on Optimization, vol. 12, no. 4, pp. 979-1006, 2002.

[14] N.J. Fliege, Multirate Digital Signal Processing: Multirate systems, filter banks, wavelets, John Wiley \& Sons, Chichester, 1994.

[15] S. Verdu, Multiuser detection. Cambridge University Press: Press Syndicate of the University of Cambridge, 1998.

[16] T. Hunziker, Z. Ju, and D. Dahlhaus, "Time-frequency channel parameterization with application to multi-mode receivers," IEICE Trans. Commun., pp. 3717-3725, Dec 2009. 